H. Katsurada

Nagoya Math. J.

Vol. 146 (1997), 199-223

\title{
AN EXPLICIT FORMULA FOR THE FOURIER COEFFICIENTS OF SIEGEL-EISENSTEIN SERIES OF DEGREE 3
}

\author{
HIDENORI KATSURADA ${ }^{1}$
}

\begin{abstract}
Using an induction formula of local densities by Kitaoka, we give an explicit formula for the Fourier coefficients of Siegel Eisenstein series of degree 3 .
\end{abstract}

\section{$\S 1$. Introduction}

Let $k$ be an even integer such that $k \geq n+2$ and

$$
E_{k}(Z)=\sum_{\{C, D\}}|C Z+D|-k .
$$

Siegel Eisenstein series of degree $n$ and of weight $k$, where $\{C, D\}$ runs over all representatives of the equivalence classes of coprime symmetric pairs of degree $n$. Then $E_{k}(Z)$ has the following Fourier expansion:

$$
E_{k}(Z)=\sum_{C} c_{k}(C) \exp (2 \pi i \operatorname{Tr}(C Z))
$$

where $C$ runs over all semi-positive definite half-integral matrices of degree $n$ over $\mathbf{Z}$, and $\operatorname{Tr}$ denotes the trace.

The Fourier coefficient of Siegel-Eisenstein series is one of the most important subjects in number theory, and many contributions have been done to it. But we have no explicit formula for it except for a few cases. The case of degree 1 is well known. In [Ma1], [Ma2], Maaß gave an explicit formula for the case of degree 2. In [Ki2] Kitaoka essentially gave an explicit formula for $c_{k}(B)$ when $n=3$ and $B$ is $\mathbf{Z}_{2}$-maximal using his recursion formula in [Ki1] (for the definition of $\mathbf{Z}_{2}$-maximal see Section 2). Partial results for the case where $B$ is not $\mathbf{Z}_{2}$-maximal were given in [O-W].

\footnotetext{
Received September 20, 1995.

${ }^{1}$ Partially supported by Grant-in-Aid for scientific Research, the Ministry of Education, Science, Sports and Culture, Japan
} 
In this paper, using the recursion formula in [Ki1] we give an explicit formula for $c_{k}(C)$ for any positive definite half-integral matrix $C$ of degree 3 . To state our main result explicitly, we introduce the notion of the "scheme" of a symmetric matrix of degree 3 with entries in the field $\mathbf{Q}_{p}$ of $p$-adic numbers. For a commutative ring $R$, let $M_{m n}(R)$ be the set of $(m, n)$-matrices with entries in $R, G L_{n}(R)$ the group of invertible elements in $M_{n n}(R)$, and $S_{n}(R)$ the set of symmetric matrices of degree $n$ with entries in $R$. Further for an integral domain $R$, let $\mathcal{H}_{n}(R)$ denote the set of half-integral matrices of degree $n$ over $R$, that is, $\mathcal{H}_{n}(R)$ is the set of symmetric matrices $\left(a_{i j}\right)$ of degree $n$ with entries in the quotient field of $R$ such that $a_{i i}(i=1, \ldots, n)$ and $2 a_{i j}(1 \leq i \neq j \leq n)$ belong to $R$. We note that $\mathcal{H}_{n}(R)=S_{n}(R)$ if $R$ contains the inverse of 2. Let $A, B$ be elements of $S_{n}(R)$. We say $A$ is equivalent to $B$ over $R$ if we have $B={ }^{t} T A T$ for some $T \in G L_{n}(R)$, and write $A \cong B$.

For two square matrices $X$ and $Y$ we write $X \perp Y=\left(\begin{array}{cc}X & 0 \\ Y & 0\end{array}\right)$. Let $\mathbf{Z}_{p}$ be the ring of $p$-adic integers, and $\mathbf{Z}_{p}^{*}$ the group of $p$-adic units. Let $p \neq 2$; then for a non-zero element $a=p^{r} c \in \mathbf{Q}_{p}$ with $r \in \mathbf{Z}, c \in \mathbf{Z}_{p}^{*}$ define

$$
\chi_{p}(a)=\left(\frac{c}{p}\right) \text { or } 0
$$

according as $r$ is even or odd. Here $\left(\frac{-}{p}\right)$ is the quadratic residue symbol modulo $p$. Let $p=2$; for a non-zero element $a=2^{r} c \in \mathbf{Q}_{2}$ with $r \in \mathbf{Z}$, $c \in \mathbf{Z}_{2}^{*}$ put

$$
\chi_{2}(a)=\left\{\begin{aligned}
+1 & \text { if } r \equiv 0 \bmod 2, c \equiv 1 \bmod 8 \\
-1 & \text { if } r \equiv 0 \bmod 2, c \equiv 5 \bmod 8 \\
0 & \text { otherwise. }
\end{aligned}\right.
$$

Further let $(,)_{p}$ denote the Hilbert symbol over $\mathbf{Q}_{p}$.

For a non-degenerate symmetric matrix $B$ of degree 3 with entries in $\mathbf{Q}_{p}$ we define five invariants $m_{1 p}(B), m_{2 p}(B), m_{3 p}(B), \eta_{p}(B), \xi_{p}(B)$ of $B$ as follows. As is well known, if $p=2, B$ has one of the following Jordan decompositions:

(1) $2^{s} b_{1} \perp 2^{t} K$ with $b_{1} \in \mathbf{Z}_{2}^{*}, K=\left(\begin{array}{cc}0 & 1 / 2 \\ 1 / 2 & 0\end{array}\right)$ or $\left(\begin{array}{cc}1 & 1 / 2 \\ 1 / 2 & 1\end{array}\right)$ and $s \geq t$ 
(2) $2^{s} K \perp 2^{t} b_{3}$ with $b_{3} \in \mathbf{Z}_{2}^{*}, K=\left(\begin{array}{cc}0 & 1 / 2 \\ 1 / 2 & 0\end{array}\right)$ or $\left(\begin{array}{cc}1 & 1 / 2 \\ 1 / 2 & 1\end{array}\right)$ and $s \geq t+2$,

(3) $2^{r} b_{1} \perp 2^{s} b_{2} \perp 2^{t} b_{3}$ with $b_{1}, b_{2}, b_{3} \in \mathbf{Z}_{2}^{*}$, and $r \geq s \geq t$,

and if $p \neq 2, B$ has the following Jordan decomposition:

(4) $p^{r} b_{1} \perp p^{s} b_{2} \perp p^{t} b_{3}$ with $r \geq s \geq t, b_{1}, b_{2}, b_{3} \in \mathbf{Z}_{p}^{*}$.

We note that we here arrange the Jordan components of $B$ in a different order from the one in [Ki4 Chap. 5]. Further we remark that the matrix $B$ in (1) $\sim(4)$ is half-integral over $\mathbf{Z}_{2}$ if and only if we have $t \geq 0$.

First let $B$ be of type (1). Then we put

$$
m_{12}(B)=t, \quad m_{22}(B)=2 t, \quad m_{32}(B)=s+2 t
$$

and

$$
\eta_{2}(B)=\left(\chi_{2}(-\operatorname{det} K)\right)^{s-t} .
$$

Further we define $\xi_{2}(B)$ by

$$
\xi_{2}(B)=\chi_{2}(-\operatorname{det} K) \text { or } 1
$$

according as $s \geq t+1$ or not.

Next let $B$ be of type (2). Then put

$$
\begin{aligned}
& m_{12}(B)=t, m_{22}(B)=t+s+2, m_{32}(B)=t+2 s \\
& \eta_{2}(B)=\left(\chi_{2}(-\operatorname{det} K)\right)^{s-t}
\end{aligned}
$$

and

$$
\xi_{2}(B)=1 .
$$

Thirdly let $B$ be of type (3). Then we put

$$
m_{12}(B)=t, \quad m_{22}(B)=s+t+2, \quad m_{32}(B)=r+s+t+2,
$$

and

$$
\eta_{2}(B)=\left(-2^{r-t} b_{1} b_{3},-2^{s-t} b_{2} b_{3}\right)_{2} .
$$

Further we define $\xi_{2}(B)$ by

$$
\xi_{2}(B)=\chi_{2}\left(-2^{s-t} b_{2} b_{3}\right), \quad \chi_{2}\left(-2^{s-t} b_{2} b_{3}\right)^{2}, \text { or } 1
$$


according as $r \geq s+3, r=s+2$, or not.

Finally let $B$ be of type (4). Then we put

$$
m_{1 p}=t, \quad m_{2 p}=s+t, \quad m_{3 p}=r+s+t
$$

and

$$
\eta_{p}(B)=\left(-p^{r-t} b_{1} b_{3},-p^{s-t} b_{2} b_{3}\right)_{p}
$$

Further we define $\xi_{p}(B)$ by

$$
\xi_{p}(B)=\chi_{p}\left(-p^{s-t} b_{2} b_{3}\right) \text { or } \chi_{p}\left(-p^{s-t} b_{2} b_{3}\right)^{2}
$$

according as $r \geq s+1$ or $r=s$.

If $p \neq 2$, by the uniqueness of Jordan decomposition these five quantities $m_{1 p}(B), m_{2 p}(B), m_{3 p}(B), \eta_{p}(B), \xi_{p}(B)$ are clearly invariants of $B$. If $p=2$, the Jordan decomposition of $B$ is not necessarily unique. However by easy observation, we see these quantities are also invariants of $B$. We remark that we can define these invariants in more intrinsic way. In fact, we have

$$
\begin{gathered}
m_{1 p}(B)=\min _{1 \leq i \leq j \leq 3} \operatorname{ord}\left(2^{1-\delta_{i j}} b_{i j}\right), \quad m_{2 p}(B)=\min _{1 \leq i \leq j \leq 3} \operatorname{ord}\left(2^{3-\delta_{i j}} B_{i j}\right) \\
m_{3 p}(B)=\operatorname{ord}(4 \operatorname{det} B)
\end{gathered}
$$

and

$$
\eta_{p}(B)=(-1)^{\delta_{2 p}} h_{p}(B)
$$

where ord denotes the normalized $p$-adic order of $\mathbf{Q}_{p}, \delta_{i j}$ is Kronecker delta, $B_{i j}$ is the $(i, j)$-th cofactor of $\operatorname{det} B$, and $h_{p}(B)$ is the Hasse invariant of $B$ (for the definition of Hasse invariant, see [Ki4, Chap. 3]). Similarly the invariant $\xi_{p}(B)$ can be also defined without using Jordan decomposition. But to make a calculation smooth we adopt the above definitions. The 5tuple $\left(m_{1 p}(B), m_{2 p}(B), m_{3 p}(B), \eta_{p}(B), \xi_{p}(B)\right)$ of invariants of $B$ is called the scheme of $B$.

Now let $B$ be a non-degenerate half-integral matrix over $\mathbf{Z}$ of degree 3 . 
Then for each prime number $p$ and integer $k$ we define $F_{p, k}(B)$ by

$$
\begin{aligned}
& F_{p, k}(B)=\sum_{i=0}^{m_{1}}\left(\sum_{j=0}^{\left[\left(m_{2}-\delta_{2 p}-1\right) / 2\right]-i} p^{(5-2 k) j}\right) p^{(3-k) i} \\
& +\eta_{p}(B) p^{(2-k) m_{3}-(3-2 k)\left(\left[m_{2} / 2\right]-\delta_{2 p}\right)} \sum_{i=0}^{m_{1}}\left(\sum_{j=n^{\prime}}^{\left[m_{2} / 2\right]-\delta_{2 p}-i} p^{(3-2 k) j}\right) p^{(2-k) i} \\
& \quad+p^{(5-2 k)\left[m_{2} / 2\right]-(2-k) m_{1}} \sum_{i=0}^{m_{3}-2 m_{2}+m_{1}} p^{(2-k) i} \xi_{p}(B)^{i+2} \sum_{j=0}^{m_{1}} p^{(2-k) j}
\end{aligned}
$$

where $m_{i}=m_{i p}(B)(i=1,2,3)$ and $n^{\prime}=n_{p}^{\prime}(B)$ is the number defined by

$$
n_{p}^{\prime}(B)= \begin{cases}1 \quad \text { if } p \neq 2 \text { and } m_{2} \equiv 0 \bmod 2 \\ \text { or if } p=2, m_{3}-2 m_{2}+m_{1}=-4, \text { and } m_{2} \equiv 0 \bmod 2 \\ 0 \quad \text { otherwise. }\end{cases}
$$

We easily see

$$
n_{p}^{\prime}(B)= \begin{cases}1 & \text { if } p \neq 2 \text { and } B \cong p^{r} b_{1} \perp p^{s} b_{2} \perp p^{t} b_{3} \text { with } b_{1}, b_{2}, b_{3} \in \mathbf{Z}_{p}^{*} \\ & r \geq s \geq t \text { and } s \equiv t \bmod 2 \\ & \text { or if } p=2 \text { and } B \cong 2^{s} K \perp 2^{t} b_{3} \text { with } K=H \text { or } Y \\ & b_{3} \in \mathbf{Z}_{2}^{*}, s \geq t+2 \text { and } s \equiv t \bmod 2 \\ 0 & \text { otherwise. }\end{cases}
$$

Remark that the set $\left\{F_{p, k}(B)\right\}_{p}$ is a genus invariant of $B$ as explained above. Further remark that $F_{p, k}(B)$ is expressed explicitly as a polynomial of $p^{-k}$ of degree $m_{3 p}(B)$, and in particular it is 1 for almost all $p$. Then our main result in this paper is

TheOREM 1.1. Let $B$ be a positive definite half-integral matrix over $\mathbf{Z}$ of degree 3 . Then we have

$$
c_{k}(B)=\frac{(-1)^{k / 2} 2^{5-k} k(k-1)(\operatorname{det} 2 B)^{k-2}}{\left|B_{k} B_{2 k-2}\right|} \prod_{p \mid 4 \operatorname{det} B} F_{p, k}(B),
$$

where $B_{i}$ is the $i$-th Bernoulli number (for the definition of Bernoulli numbers see, for example, Miyake [Mi, Chap. 3]).

It should be remarked that $c_{k}(B)$ can be completely determined by the set $\left\{\left(m_{1 p}(B), m_{2 p}(B), m_{3 p}(B), \eta_{p}(B), \xi_{p}(B)\right)\right\}_{p}$ of schemes of $B$. To state the 
outline of the proof, for a half-integral matrix $S$ of degree $m$ and symmetric matrix $T$ of degree $n$ with entries in $\mathbf{Q}_{p}$, put

$$
\begin{aligned}
& \mathcal{A}_{e}(T, S)=\#\left\{X \in M_{m n}\left(\mathbf{Z}_{p}\right) / p^{e} M_{m n}\left(\mathbf{Z}_{p}\right) ; S[X]-T \in p^{e} \mathcal{H}_{n}\left(\mathbf{Z}_{p}\right)\right\} \\
& \mathcal{B}_{e}(T, S)=\#\left\{X \in \mathcal{A}_{e}(T, S) ; X \text { has the maximal rank } \bmod p\right\}
\end{aligned}
$$

and

$$
\begin{aligned}
& \alpha_{p}(T, S)=\lim _{e \rightarrow \infty} p^{(-m n+n(n+1) / 2) e} \mathcal{A}_{e}(T, S), \\
& \beta_{p}(T, S)=\lim _{e \rightarrow \infty} p^{(-m n+n(n+1) / 2) e} \mathcal{B}_{e}(T, S)
\end{aligned}
$$

where for two matrices $T, X, T[X]$ denotes ${ }^{t} X T X$. Let $H_{k}=\overbrace{H \perp \ldots \perp H}^{k}$ with $H=\left(\begin{array}{cc}0 & 1 / 2 \\ 1 / 2 & 0\end{array}\right)$. Then it follows from Maaß [Ma1] that for a positive definite half-integral matrix $B$ of degree 3 , we have

$$
c_{k}(B)=(-1)^{k / 2} 2^{3(k-1)}(\operatorname{det} B)^{k-2} \prod_{i=0}^{2} \frac{\pi^{k-i / 2}}{\Gamma(k-i / 2)} \prod_{p} \alpha_{p}\left(B, H_{k}\right),
$$

where $\Gamma(z)$ is the gamma function, and $p$ runs over all prime numbers. Thus our main theorem can be reduced to the following.

THEOREM 1.2. Let the notation and the assumption be as above. Then for any non-degenerate half-integral symmetric matrix $B$ of degree 3 over $\mathbf{Z}_{p}$ we have

$$
\alpha_{p}\left(B, H_{k}\right)=\left(1-p^{-k}\right)\left(1-p^{2-2 k}\right) F_{p, k}(B)
$$

Theorem 1.2 for the case $p \neq 2$ has been treated by Kitaoka in [Ki2] though it has not been formulated in the above form (cf. Section 4). The value $\alpha_{2}\left(B, H_{k}\right)$ for a $\mathbf{Z}_{2}$-maximal matrix $B$ of degree 3 is well known (cf. Proposition 2.2). Thus Kitaoka essentially proved Theorem 1.1 in this case. The crutial part of this paper is the proof for the case $p=2$. The method we adopt is similar to that in [Ki2], but we give a more concise formula than that by introducing the notion of the scheme. Fixing $\eta_{p}(B)$ and $\xi_{p}(B)$ we temporarily write $A_{p}\left(m_{1}, m_{2}, m_{3}\right)$ for $\alpha_{p}\left(B, H_{k}\right)$. Then first we give an explicit formula for $A_{p}\left(0, m_{2}, m_{3}\right)$ when $m_{2}=0,1,2 \delta_{2 p}$ or $2 \delta_{2 p}+1$ (cf. Theorem 3.1.3). Next we express $A_{p}\left(0, m_{2}, m_{3}\right)$ using $A_{p}\left(0, m_{2}-\right.$ 
$\left.2\left[\left(m_{2}-2 \delta_{2 p}\right) / 2\right], m_{3}-4\left[\left(m_{2}-2 \delta_{2 p}\right) / 2\right]\right)$ by a certain induction formula when $m_{2} \geq 2 \delta_{2 p}$ (cf. Theorem 3.2.1). Thirdly we give an induction formula by which we can express $A_{p}\left(m_{1}, m_{2}, m_{3}\right)$ using $A_{p}\left(0, m_{2}-2 m_{1}, m_{3}-3 m_{1}\right)$ (cf. Theorem 3.3.1), and finally combining these results, we complete the proof. The proof is rather long but comprehensible, and our mehtod is applicalble to Siegel-Eisenstein series of any degree, and a lot of information on the Fourier cofficients of it can be obtained. Further we remark that the same result in this paper can be obtained by using a modification of our recursion formula in [Ka1] and [Ka2]. In fact, in the first draft of this paper we have obtained the main result using it. The detail will be published in a subsequent paper. We also remark that D. Zagier conjectured some induction formulae similar to ours. The author thanks Professors Y. Kitaoka and T. Ibukiyama for many helpful discussions and suggestions. He also thanks the referee for valuable comments.

\section{$\S 2$. Kitaoka's recursion formula}

In this section we review Kitaoka's recursion formula for local densities following [Ki1]. Put

$$
\begin{gathered}
f_{1}=\left(\begin{array}{ccc}
p^{-1} & 0 & 0 \\
0 & 1 & 0 \\
0 & 0 & 1
\end{array}\right), f_{2}(\alpha)=\left(\begin{array}{ccc}
1 & p^{-1} \alpha & 0 \\
0 & p^{-1} & 0 \\
0 & 0 & 1
\end{array}\right) \quad(\alpha=0, \ldots, p-1), \\
f_{3}(\alpha, \beta)=\left(\begin{array}{ccc}
1 & 0 & p^{-1} \alpha \\
0 & 1 & p^{-1} \beta \\
0 & 0 & p^{-1}
\end{array}\right) \quad(\alpha, \beta=0,1, \ldots, p-1) \\
g_{1}=\left(\begin{array}{ccc}
p^{-1} & 0 & 0 \\
0 & p^{-1} & 0 \\
0 & 0 & 1
\end{array}\right), g_{2}(\alpha)=\left(\begin{array}{ccc}
p^{-1} & 0 & 0 \\
0 & 1 & p^{-1} \alpha \\
0 & 0 & p^{-1}
\end{array}\right), \\
g_{3}(\alpha, \beta)=\left(\begin{array}{ccc}
1 & p^{-1} \alpha & p^{-1} \beta \\
0 & p^{-1} & 0 \\
0 & 0 & p^{-1}
\end{array}\right) .
\end{gathered}
$$

Further put

$$
\mathcal{F}=\left\{f_{1}, f_{2}(\alpha)(\alpha=0,1, \ldots, p-1), f_{3}(\alpha, \beta)(\alpha, \beta=0, \ldots, p-1)\right\}
$$


and

$$
\mathcal{G}=\left\{g_{1}, g_{2}(\alpha)(\alpha=0,1, \ldots, p-1), g_{3}(\alpha, \beta)(\alpha, \beta=0, \ldots, p-1)\right\} .
$$

Then by [Ki1, Theorem 1] we have

TheOREM 2.1. Let $B$ be a non-degenerate half-integral matrix of degree 3 over $\mathbf{Z}_{p}$. Then we have

$$
\begin{aligned}
\alpha_{p}\left(B, H_{k}\right)=p^{4-2 k} \sum_{f \in \mathcal{F}} \alpha_{p}(B[f] & \left.H_{k}\right)-p^{9-4 k} \sum_{g \in \mathcal{G}} \alpha_{p}\left(B[g], H_{k}\right) \\
& +p^{15-6 k} \alpha_{p}\left(B\left[p^{-1} E_{3}\right], H_{k}\right)+\beta_{p}\left(B, H_{k}\right),
\end{aligned}
$$

where $E_{3}$ denotes the unit matrix of degree 3 .

For two symmetric matrices $B$ and $B^{\prime}$ of degree $n$ with entries in $\mathbf{Q}_{p}$, we write $B^{\prime}<B$ if there exists a non-degenerate non-unimodular matrix $X$ of degree $n$ with entries in $\mathbf{Z}_{p}$ such that $B^{\prime}=B[X]$. This defines an order in $S_{n}\left(\mathbf{Q}_{p}\right)$. We call $B \mathbf{Z}_{p}$-maximal or simply maximal if it is maximal in $\mathcal{H}_{3}\left(\mathbf{Z}_{p}\right)$. From now on we simply write $\alpha_{p}(B)$ and $\beta_{p}(B)$ instead of $\alpha_{p}\left(B, H_{k}\right)$ and $\beta_{p}\left(B, H_{k}\right)$, respectively, and put

$$
d=d_{p}=\left(1-p^{-k}\right)\left(1-p^{2-2 k}\right) .
$$

The following proposition is well known.

Proposition 2.2. (1) Let $H$ be the matrix defined in Section 1, and $Y=\left(\begin{array}{cc}1 & 1 / 2 \\ 1 / 2 & 1\end{array}\right)$. Then a maximal matrix $B$ in $\mathcal{H}_{3}\left(\mathbf{Z}_{2}\right)$ is equivalent, over $\mathbf{Z}_{2}$, to one of the following forms:

$$
\begin{gathered}
2^{r} b_{1} \perp K, \text { with } r=0,1, K=H \text { or } Y, b_{1} \in \mathbf{Z}_{2}^{*}, \\
\qquad b_{1} \perp 2 Y \text { with } b_{1} \in \mathbf{Z}_{2}^{*}
\end{gathered}
$$

(2) Let $p \neq 2$. Then a maximal matrix $B$ in $\mathcal{H}_{3}\left(\mathbf{Z}_{p}\right)$ has one of the following Jordan forms:

$$
\begin{gathered}
p^{r} b_{1} \perp b_{2} \perp b_{3} \text { with } r=0,1, b_{1}, b_{2}, b_{3} \in \mathbf{Z}_{p}^{*}, \\
p b_{1} \perp p b_{2} \perp b_{3} \text { with } b_{1}, b_{2}, b_{3} \in \mathbf{Z}_{p}^{*},-b_{1} b_{2} \notin \mathbf{Z}_{p}^{* 2} .
\end{gathered}
$$

Further in (1) and (2), for such a matrix $B$ we have

$$
\alpha_{p}(B)=\beta_{p}(B)
$$


Remark. Let $b_{1}, b_{2}, b_{3}$ be elements of $\mathbf{Z}_{2}^{*}$, and $K=H$ or $Y$. Then we have

$$
2^{r}\left(b_{1} \perp b_{2} \perp b_{3}\right) \cong 2^{r} b_{1}^{\prime} \perp 2^{r+1} K^{\prime}
$$

where $b_{1}^{\prime} \in \mathbf{Z}_{2}^{*}$, and $K^{\prime}=H$ or $Y$ according as $\eta_{2}\left(2^{r}\left(b_{1} \perp b_{2} \perp b_{3}\right)\right)=1$ or -1 .

Now by [Ki3, Lemma 9] we have

Proposition 2.3. Let $B$ be a non-degenerate half-integral matrix of degree 3 over $\mathbf{Z}_{p}$, and $m_{1 p}(B)$ and others be the invariants of $B$ defined in Section 1. Then we have

$$
\beta_{p}(B)=\left\{\begin{array}{lll}
d & \text { if } m_{1 p}(B)=m_{2 p}(B)=m_{3 p}(B)=0 \\
d\left(1+p^{2-k} \xi_{p}(B)\right) & \text { if } m_{1 p}(B)=m_{2 p}(B)=0 \\
d\left(1-p^{4-2 k}\right) & \text { if } m_{1 p}(B)=0, m_{2 p}(B) \geq 1, \\
& \text { and } m_{3 p}(B) \geq 1 \\
d\left(1-p^{4-2 k}\right)\left(1+p^{3-k}\right) & \text { if } m_{1 p}(B) \geq 1 &
\end{array}\right.
$$

Corollary. Let $B$ and $B^{\prime}$ be two half-integral matrices of degree 3 over $\mathbf{Z}_{p}$ with the same scheme. Then we have

$$
\beta_{p}(B)=\beta_{p}\left(B^{\prime}\right)
$$

\section{$\S 3$. Proof of the main result}

In this section we prove Theorem 1.2. Let $d=\left(1-p^{-k}\right)\left(1-p^{2-2 k}\right)$ be as stated in Section 2. Put $\Gamma=G L_{3}\left(\mathbf{Z}_{p}\right)$, and for two elements $f, g$ of $G L_{3}\left(\mathbf{Q}_{p}\right)$ we write

$$
f \equiv g \bmod \Gamma
$$

if we have $f=g h$ with some $h \in \Gamma$. Further we write $\mathcal{E}=\mathcal{F} \cup \mathcal{G} \cup\left\{p^{-1} E_{3}\right\}$, where $\mathcal{F}$ and $\mathcal{G}$ are the subsets of $G L_{3}\left(\mathbf{Q}_{p}\right)$ defined in Section 2.

\subsection{First step}

LemMA 3.1.1. (1) Let $p=2$. Let $b_{1}, b_{2}, b_{3} \in \mathbf{Z}_{2}^{*}$ and $K=H$ or $Y$.

(1.1) Let $B=b_{1} \perp b_{2} \perp b_{3}$ or $B=2 K \perp b_{3}$. Then we have

$$
\alpha_{2}(B)=d\left(1+\eta_{2}(B) 2^{4-2 k}\right) .
$$

(1.2) Let $B=2 b_{1} \perp b_{2} \perp b_{3}$. Then we have

$$
\alpha_{2}(B)=d\left(1+\eta_{2}(B) 2^{6-3 k}\right)
$$


(1.3) Let $B=2 b_{1} \perp 2 b_{2} \perp b_{3}$. Then we have

$$
\alpha_{2}(B)=d\left(1+\eta_{2}(B) 2^{8-4 k}\right) .
$$

(2) Let $p \neq 2$ and $B=p b_{1} \perp p b_{2} \perp b_{3}$ with $b_{1}, b_{2}, b_{3} \in \mathbf{Z}_{p}^{*}$. Then we have

$$
\alpha_{p}(B)=d\left(1+\eta_{p}(B) p^{4-2 k}\right) .
$$

Proof. First let $B$ be as in (1.1). If we have $\eta_{2}(B)=-1$, the assertion (1.1) for this case holds by (1) of Proposition 2.2, the remark after Proposition 2.2 and Proposition 2.3. If we have $\eta_{2}(B)=1$, again by the remark after Proposition 2.2, $B$ is equivalent to $b_{1}^{\prime} \perp 2 H$ with $b_{1}^{\prime} \in \mathbf{Z}_{2}^{*}$. Then by Theorem 2.1 we have

$$
\alpha_{2}(B)=2^{5-2 k} \alpha_{2}\left(b_{1}^{\prime} \perp H\right)+\beta_{2}(B) .
$$

Thus the assertion (1.1) holds again by (1) of Proposition 2.2 and Proposition 2.3. The other assertions can be similarly proved.

Lemma 3.1.2. (1) Let $p=2$. Let $b_{1}, b_{2}, b_{3} \in \mathbf{Z}_{2}^{*}$, and $K=H$ or $Y$.

(1.1) Let $r \geq 0$, and put $B=2^{r} b_{1} \perp K$. Then we have

$$
\alpha_{2}(B)=d \sum_{i=0}^{r}\left(\chi_{2}(-\operatorname{det} K) 2^{2-k}\right)^{i} .
$$

(1.2) Let $r \geq s, s=0,1$. Further assume that $-b_{2} b_{3} \not \equiv 1 \bmod 4$ if $s=0$. Put $B=2^{r} b_{1} \perp 2^{s} b_{2} \perp b_{3}$. Then we have

$$
\alpha_{2}(B)=d\left(1+\eta_{2}(B) 2^{(\operatorname{ord}(\operatorname{det} B)+2)(2-k)}\right) .
$$

(1.3) Let $r \geq 0$, and assume that $-b_{2} b_{3} \equiv 1 \bmod 4$. Put $B=2^{r} b_{1} \perp b_{2} \perp b_{3}$. Then we have

$$
\alpha_{2}(B)=d\left(1+\eta_{2}(B) 2^{(\operatorname{ord}(\operatorname{det} B)+2)(2-k)}\right)+2^{5-2 k} d \sum_{i=0}^{r-2}\left(\chi_{2}\left(-b_{2} b_{3}\right) 2^{2-k}\right)^{i} .
$$

(2) Let $p \neq 2$. Let $b_{1}, b_{2}, b_{3} \in \mathbf{Z}_{p}^{*}$.

(2.1) Let $r \geq 1$. Put $B=p^{r} b_{1} \perp p b_{2} \perp b_{3}$. Then we have

$$
\alpha_{p}(B)=d\left(1+\eta_{p}(B) p^{\operatorname{ord}(\operatorname{det} B)(2-k)}\right) .
$$

(2.2) Let $r \geq 0$. Put $p^{r} b_{1} \perp b_{2} \perp b_{3}$. Then we have

$$
\alpha_{p}(B)=d \sum_{i=0}^{r}\left(\chi_{p}\left(-b_{2} b_{3}\right) p^{2-k}\right)^{i} .
$$


Proof. (1.1) By Theorem 2.1, for $r \geq 1$ we have

$$
\alpha_{2}(B)=2^{4-2 k} \alpha_{2}\left(2^{r-2} b_{1} \perp K\right)+\beta_{2}(B) .
$$

Thus the assertion follows from (1) of Proposition 2.2 and Proposition 2.3.

Here we give the following remark, which is a direct consequence of (1.1):

$$
\alpha_{2}\left(2^{r} b \perp K\right)-2^{4-2 k}\left(2^{r-2} b^{\prime} \perp K\right)=d\left(1+2^{2-k} \chi_{2}(-\operatorname{det} K)\right)
$$

for $r \geq 1$ and $b, b^{\prime} \in \mathbf{Z}_{2}^{*}$.

(1.2) The case $r \leq 1$ follows from Lemma 3.1.1. Assume $r \geq 2$, and $s=0$ or 1 . Then similarly to (1.1) we have

$$
\alpha_{2}(B)=2^{4-2 k} \alpha_{2}\left(2^{r-2} b_{1} \perp 2^{s} b_{2} \perp b_{3}\right)+\beta_{2}(B) .
$$

Thus the assertion can be proved by Proposition 2.3 and Lemma 3.1.1.

(1.3) Let $r \geq 2$. Then by Theorem 2.1 we have

$$
\begin{gathered}
\alpha_{2}(B)=2^{4-2 k} \alpha_{2}\left(B\left[f_{1}\right]\right)+2^{4-2 k} \sum_{\alpha=0,1} \alpha_{2}\left(B\left[f_{3}(\alpha, 1)\right]\right) \\
-2^{9-4 k} \alpha_{2}\left(B\left[g_{2}(1)\right]\right)+\beta_{2}(B) .
\end{gathered}
$$

Then we have

$$
B\left[f_{1}\right]=2^{r-2} b_{1} \perp b_{2} \perp b_{3},
$$

and

$$
B\left[g_{2}(1)\right] \cong 2^{r-2} b_{1} \perp K^{\prime},
$$

where $K^{\prime}=H$ or $Y$ according as $-b_{2} b_{3} \equiv 1 \bmod 8$ or not.

First let $r=2$. Then by easy calculation we have

$$
B\left[f_{3}(\alpha, 1)\right] \cong 4 b_{1}^{\prime}(\alpha) \perp K^{\prime}(\alpha)
$$

for $\alpha=0,1$, where $b_{1}^{\prime}(\alpha) \in \mathbf{Z}_{2}^{*}$ and $K^{\prime}(\alpha)=H$ or $Y$ according as $-b_{2} b_{3}=$ $4 \alpha+1 \bmod 8$ or $\equiv 4 \alpha+5 \bmod 8$. Thus the assertion follows from Proposition 2.3, Lemma 3.1.1, and (1.1).

Next let $r \geq 3$. Then we have

$$
B\left[f_{3}(\alpha, 1)\right] \cong 2^{r} b_{1}^{\prime}(\alpha) \perp K^{\prime}
$$


for $\alpha=0,1$ with $b_{1}^{\prime}(\alpha) \in \mathbf{Z}_{2}^{*}$. Thus the assertion follows from Proposition 2.3, Lemma 3.1.1, and $(*)$.

(2) The assertion can be proved similarly to (1) by using Theorems 2.1 and 2.4, Propositions 2.2 and 2.3, and (2) of Lemma 3.1.1.

Summarizing the above results, we have

THEOREM 3.1.3. Let $b_{1}, b_{2}, b_{3}$ and $K$ be as in Lemma 3.1.2. Let $B=$ $p^{r} b_{1} \perp p^{s} b_{2} \perp b_{3}$ with $r \geq s, 0 \leq s \leq 1$, or $2^{r} b_{1} \perp K$ with $r \geq 0$, and $B_{2}$ be $a$ symmetric matrix of degree 2 such that $B=p^{r} b_{1} \perp B_{2}$. Then we have

$$
\begin{aligned}
\alpha_{p}(B)=d p^{(5-2 k) l^{\prime}} & \sum_{i=0}^{m_{3}-2 m_{2}} \xi_{p}(B)^{i+2} p^{(2-k) i} \\
+ & \begin{cases}0 & \text { if } 4 \operatorname{det} B_{2} \text { is unit } \\
d\left(1+\eta_{p}(B) p^{m_{3}(2-k)}\right) & \text { otherwise, }\end{cases}
\end{aligned}
$$

where $l^{\prime}=0$ or $\delta_{2 p}$ according as $p=2$ and $B_{2}=K$, or not.

\subsection{Second step}

Now for a non-degenerate symmetric matrix $B_{1}$ of degree 2 with entries in $\mathbf{Q}_{p}$ and $b_{3} \in \mathbf{Z}_{p}^{*}$, put

$$
\tilde{\gamma}\left(B_{1}, b_{3}\right)=\alpha_{p}\left(p^{2} B_{1} \perp b_{3}\right)-p^{5-2 k} \alpha_{p}\left(B_{1} \perp b_{3}\right) .
$$

Now fixing an element $b_{3} \in \mathbf{Z}_{p}^{*}$ we define a subset $\mathcal{H}_{2}^{\prime}\left(\mathbf{Z}_{2}\right)\left(b_{3}\right)$ of $S_{2}\left(\mathbf{Q}_{p}\right)$ by

$$
\begin{aligned}
\mathcal{H}_{2}^{\prime}\left(\mathbf{Z}_{2}\right) & \left(b_{3}\right) \\
= & \left\{B_{1} ; B_{1} \cong 2^{r} b_{1} \perp 2^{-2} b_{2} \text { with } r \geq-1, b_{1}, b_{2} \in \mathbf{Z}_{2}^{*},-b_{2} b_{3} \not 1 \bmod 4\right\} \\
& \cup\left\{B_{1} ; B_{1} \cong 2^{r} b_{1} \perp 2^{-1} b_{2} \text { with } r \geq 0, b_{1}, b_{2} \in \mathbf{Z}_{2}^{*}\right\} \\
& \cup\left\{B_{1} ; B_{1} \cong 2^{r} b_{1} \perp 2^{r} b_{2} \text { with } r=0,-1,-2, b_{1}, b_{2} \in \mathbf{Z}_{2}^{*}\right\} \\
& \cup\left\{B_{1} ; B_{1} \cong 2^{i} K \text { with } i=0,-1, K=H, Y\right\},
\end{aligned}
$$

and by

$$
\begin{aligned}
\mathcal{H}_{2}^{\prime}\left(\mathbf{Z}_{p}\right)\left(b_{3}\right)=\left\{B_{1} ; B_{1} \cong p^{-1} b_{1} \perp p^{-2} b_{2} \text { with } b_{1}, b_{2} \in \mathbf{Z}_{p}^{*}\right\} & \\
& \cup\left\{B_{1} ; B_{1} \cong p^{r} b_{1} \perp p^{-1} b_{2} \text { with } r \geq-1, b_{1}, b_{2} \in \mathbf{Z}_{p}^{*}\right\} \\
& \cup\left\{B_{1} ; B_{1} \cong b_{1} \perp b_{2} \text { with } b_{1}, b_{2} \in \mathbf{Z}_{p}^{*}\right\}
\end{aligned}
$$

for $p \neq 2$. We note that $2^{-1} b_{1} \perp 2^{-2} b_{2}$ with $b_{1}, b_{2} \in \mathbf{Z}_{2}^{*}$ belongs to $\mathcal{H}_{2}^{\prime}\left(\mathbf{Z}_{2}\right)\left(b_{3}\right)$ even if $-b_{2} b_{3} \equiv 1 \bmod 4$, and $\mathcal{H}_{2}^{\prime}\left(\mathbf{Z}_{p}\right)\left(b_{3}\right)$ is independent of $b_{3}$ if $p \neq 2$. 
TheOREM 3.2.1. Let $b_{3} \in \mathbf{Z}_{p}^{*}$ and $B_{1}$ an element of $\mathcal{H}_{2}\left(\mathbf{Z}_{p}\right) \cup$ $\mathcal{H}_{2}^{\prime}\left(\mathbf{Z}_{p}\right)\left(b_{3}\right)$. Put $B=B_{1} \perp b_{3}$. Then we have

$$
\tilde{\gamma}\left(B_{1}, b_{3}\right)=d\left(1+\eta_{p}(B) p^{\left(m_{3}(B)+4\right)(2-k)}\right) .
$$

To prove the theorem, we need some preliminary result.

Proposition 3.2.2. The assertion in Theorem 3.2.1 holds for $b_{3} \in \mathbf{Z}_{p}^{*}$ and $B_{1} \in \mathcal{H}_{2}^{\prime}\left(\mathbf{Z}_{p}\right)\left(b_{3}\right)$.

Proof. First let $p=2$. If $B_{1} \not K$ and $B_{1} \not b_{1} \perp b_{2}$ the assertion follows immediately from (1) of Lemma 3.1.1 and (1.2) of Lemma 3.1.2 remarking that $\alpha_{2}\left(B_{1} \perp b_{3}\right)=0$. Next let $B_{1}=K$. Put

$$
f_{1}^{\prime}=\left(\begin{array}{cc}
2^{-1} & 0 \\
0 & 1
\end{array}\right), \quad f_{2}^{\prime}(\alpha)=\left(\begin{array}{cc}
1 & 2^{-1} \alpha \\
0 & 2^{-1}
\end{array}\right)(\alpha=0,1)
$$

and

$$
\mathcal{X}_{2}^{\prime}=\left\{f_{1}^{\prime}, f_{2}^{\prime}(0), f_{2}^{\prime}(1)\right\}
$$

Then by Theorem 2.1 we have

$$
\begin{aligned}
\alpha_{2}\left(4 B_{1} \perp b_{3}\right) & =2^{4-2 k} \sum_{X \in \mathcal{X}_{2}^{\prime}} \alpha_{2}\left(4 B_{1}[X] \perp b_{3}\right)-2^{9-4 k} \alpha_{2}\left(B_{1} \perp b_{3}\right)+\beta_{2}\left(4 B_{1} \perp b_{3}\right), \\
\alpha_{2}\left(B_{1} \perp b_{3}\right) & =\beta_{2}\left(B_{1} \perp b_{3}\right)=d,
\end{aligned}
$$

and

$$
\beta_{2}\left(4 B_{1} \perp b_{3}\right)=d\left(1-2^{4-2 k}\right) .
$$

Remark that $B_{1}[X] \in \mathcal{H}_{2}^{\prime}\left(\mathbf{Z}_{2}\right)\left(b_{3}\right)$ but

$$
\alpha_{2}\left(B_{1}[X] \perp b_{3}\right)=0
$$

for any $X \in \mathcal{X}_{2}^{\prime}$. Thus we have

$$
\tilde{\gamma}\left(B_{1}, b_{3}\right)=2^{4-2 k} \sum_{X \in \mathcal{X}_{2}^{\prime}} \tilde{\gamma}\left(B_{1}[X], b_{3}\right)+d\left(1-2^{4-2 k}\right) .
$$

Thus the assertion follows from the above. Similarly the assertion holds for $B_{1}=b_{1} \perp b_{2}$. The assertion can also be proved for $p \neq 2$ by using (2) of Lemmas 3.1.1, and 3.1.2. 
Proof of Theorem 3.2.1. First let $p=2$. Let " $<$ " be the order in $\mathcal{H}_{2}\left(\mathbf{Z}_{2}\right) \cup \mathcal{H}_{2}^{\prime}\left(\mathbf{Z}_{2}\right)\left(b_{3}\right)$ defined in Section 2 . We prove the assertion by induction on this order. Clearly all maximal matrices in $\mathcal{H}_{2}\left(\mathbf{Z}_{2}\right) \cup \mathcal{H}_{2}^{\prime}\left(\mathbf{Z}_{2}\right)\left(b_{3}\right)$ belong to $\mathcal{H}_{2}^{\prime}\left(\mathbf{Z}_{2}\right)\left(b_{3}\right)$. Thus the assertion holds for all maximal matrices $B_{1} \in \mathcal{H}_{2}\left(\mathbf{Z}_{2}\right) \cup \mathcal{H}_{2}^{\prime}\left(\mathbf{Z}_{2}\right)\left(b_{3}\right)$ by Proposition 3.2.2. Assume that $B_{1}$ is not maximal in $\mathcal{H}_{2}\left(\mathbf{Z}_{2}\right)\left(b_{3}\right) \cup \mathcal{H}_{2}^{\prime}\left(\mathbf{Z}_{2}\right)\left(b_{3}\right)$ and that the assertion holds for any $B_{1}^{\prime}$ such that $B_{1}<B_{1}^{\prime}$. In view of Proposition 3.2 .2 we may assume that $B_{1} \notin \mathcal{H}_{2}^{\prime}\left(\mathbf{Z}_{2}\right)\left(b_{3}\right)$; then we have

$$
B_{1} \cong 2^{r} b_{1} \perp 2^{s} b_{2}(r>s \geq 0), \cong 2^{r} b_{1} \perp 2^{r} b_{2}(r \geq 1) \text { or } \cong 2^{r} K(r \geq 1) .
$$

First let $B_{1}=2^{r} b_{1} \perp b_{2}$ with $r>0,-b_{2} b_{3} \equiv 1 \bmod 4$. Then by Theorem 2.1 we have

$$
\begin{array}{r}
\alpha_{2}\left(4 B_{1} \perp b_{3}\right)=2^{4-2 k} \alpha_{2}\left(\left(4 B_{1} \perp b_{3}\right)\left[f_{1}\right]\right)+2^{4-2 k} \sum_{\alpha=0,1} \alpha_{2}\left(\left(4 B_{1} \perp b_{3}\right)\left[f_{2}(\alpha)\right]\right) \\
-2^{9-4 k} \alpha_{2}\left(\left(4 B_{1} \perp b_{3}\right)\left[g_{1}\right]\right)+\beta_{2}\left(4 B_{1} \perp b_{3}\right)
\end{array}
$$

and

$$
\begin{aligned}
\alpha_{2}\left(B_{1} \perp b_{3}\right)=2^{4-2 k} \alpha_{2}\left(\left(B_{1} \perp b_{3}\right)\left[f_{1}\right]\right)+2^{4-2 k} \sum_{\alpha=0,1} \alpha_{2}\left(\left(B_{1} \perp b_{3}\right)\left[f_{3}(\alpha, 1)\right]\right) \\
-2^{9-4 k} \alpha_{2}\left(\left(B_{1} \perp b_{3}\right)\left[g_{2}(1)\right]\right)+\beta_{2}\left(B_{1} \perp b_{3}\right) .
\end{aligned}
$$

Clearly we have

$$
\left(4^{i} B_{1} \perp b_{3}\right)\left[f_{1}\right]=4^{i} B_{1}\left[f_{1}^{\prime}\right] \perp b_{3}(i=0,1),
$$

where $f_{1}^{\prime}$ is the matrix defined in the proof of Proposition 3.2.2. For $\alpha=0$, 1 , put $\tilde{B}(\alpha)=\left(4 B_{1} \perp b_{3}\right)\left[f_{2}(\alpha)\right]$. Then clearly we have

$$
\tilde{B}(\alpha)\left[f_{1}\right] \cong\left(4 B_{1} \perp b_{3}\right)\left[g_{1}\right]=B_{1} \perp b_{3} .
$$

Thus we have

$$
\begin{aligned}
& \tilde{\gamma}\left(B_{1}, b_{3}\right)=2^{4-2 k} \tilde{\gamma}\left(B_{1}\left[f_{1}^{\prime}\right], b_{3}\right) \\
& \quad+2^{4-2 k} \sum_{\alpha=0,1}\left\{\alpha_{2}(\tilde{B}(\alpha))-2^{4-2 k} \alpha_{2}\left(\tilde{B}(\alpha)\left[f_{1}\right]\right)-2^{4-2 k} \alpha_{2}\left(\tilde{B}(\alpha)\left[f_{3}(0,1)\right]\right)\right. \\
& \left.\quad-2^{4-2 k} \alpha_{2}\left(\tilde{B}(\alpha)\left[f_{3}(1,1)\right]\right)+2^{9-4 k} \alpha_{2}\left(\tilde{B}(\alpha)\left[g_{2}(1)\right]\right)\right\} \\
& \quad+2^{8-4 k} \sum_{\alpha=0,1}\left\{\alpha_{2}\left(\tilde{B}(\alpha)\left[f_{3}(0,1)\right]\right)+\alpha_{2}\left(\tilde{B}(\alpha)\left[f_{3}(1,1)\right]\right)\right. \\
& \quad-2^{5-2 k} \alpha_{2}\left(\tilde{B}(\alpha)\left[g_{2}(1)\right]\right)-2 \alpha_{2}\left(B\left[f_{3}(\alpha, 1)\right]\right) \\
& \left.\quad+2^{5-2 k} \alpha_{2}\left(B\left[g_{2}(1)\right]\right)\right\}+d\left(1-2^{4-2 k}\right)\left(1-2^{5-2 k}\right) .
\end{aligned}
$$


By Theorem 2.1, the second term on the right-hand side is

$$
2^{4-2 k} \sum_{\alpha=0,1} \beta_{2}(\tilde{B}(\alpha))=2^{5-2 k} d\left(1-2^{4-2 k}\right) .
$$

We claim the third term is 0 . To prove this we remark that

$$
B\left[f_{3}(0,1)\right] \cong 2^{r} b_{1} \perp K^{\prime}, \quad B\left[g_{2}(1)\right] \cong 2^{r-2} b_{1} \perp K^{\prime}, \quad \tilde{B}(0)\left[g_{2}(1)\right] \cong 2^{r} b_{1} \perp K^{\prime}
$$

and

$$
\tilde{B}(0)\left[f_{3}(\beta, 1)\right] \cong 2^{r+2} b_{1} \perp K^{\prime}
$$

for any $\beta=0,1$, where $K^{\prime}=H$ or $Y$ according as $-b_{2} b_{3} \equiv 1 \bmod 8$ or $\equiv 5 \bmod 8$. Thus by $(*)$ in the proof of Lemma 3.1.2 we have

$$
\begin{aligned}
\alpha_{2}\left(\tilde{B}(0)\left[f_{3}(0,1)\right]\right)+\alpha_{2}(\tilde{B}(0) & {\left.\left[f_{3}(1,1)\right]\right)-2^{5-2 k} \alpha_{2}\left(\tilde{B}(0)\left[g_{2}(1)\right]\right) } \\
& -2 \alpha_{2}\left(B\left[f_{3}(0,1)\right]\right)+2^{5-2 k} \alpha_{2}\left(B\left[g_{2}(1)\right]\right)=0 .
\end{aligned}
$$

On the other hand, we have

$$
\tilde{B}(1)\left[f_{3}(\beta, 1)\right] \notin \mathcal{H}_{3}\left(\mathbf{Z}_{2}\right), \cong 2^{r+2} 5 b_{1} \perp K^{\prime \prime} \text { or } \cong 2^{r+2} b_{1} \perp K^{\prime}
$$

according as $r=1,2$, or not, and

$$
\tilde{B}(1)\left[g_{2}(1)\right] \notin \mathcal{H}_{3}\left(\mathbf{Z}_{2}\right), \cong 2^{r} 5 b_{1} \perp K^{\prime \prime} \text { or } \cong 2^{r} b_{1} \perp K^{\prime}
$$

according as $r=1,2$ or not, where $K^{\prime \prime}=Y$ or $H$ according as $-b_{2} b_{3} \equiv$ $1 \bmod 8$ or $\equiv 5 \bmod 8$. Further

$$
B\left[f_{3}(1,1)\right] \notin \mathcal{H}_{3}\left(\mathbf{Z}_{2}\right), \cong 2^{r} 5 b_{1} \perp K^{\prime \prime} \text { or } \cong 2^{r} b_{1} \perp K^{\prime}
$$

according as $r=1,2$ or not, and

$$
B\left[g_{2}(1)\right] \notin \mathcal{H}_{3}\left(\mathbf{Z}_{2}\right), \text { or } \cong 2^{r-2} b_{1} \perp K^{\prime}
$$

according as $r=1$ or not. Thus again by $(*)$ we have

$$
\begin{aligned}
\alpha_{2}\left(\tilde{B}(1)\left[f_{3}(0,1)\right]\right)+\alpha_{2}(\tilde{B}(1) & {\left.\left[f_{3}(1,1)\right]\right)-2^{5-2 k} \alpha_{2}\left(\tilde{B}(1)\left[g_{2}(1)\right]\right) } \\
& -2 \alpha_{2}\left(B\left[f_{3}(1,1)\right]\right)+2^{5-2 k} \alpha_{2}\left(B\left[g_{2}(1)\right]\right)=0 .
\end{aligned}
$$

Thus the claim has been proved, and therefore, the assertion holds by the induction hypothesis. 
Next assume that $B_{1}=2^{r} b_{1} \perp 2^{s} b_{2}$ with $r \geq s>0, B_{1}=2^{r} b_{1} \perp b_{2}$ with $r>0$ and $-b_{2} b_{3} \not \equiv 1 \bmod 4$, or $B_{1}=2^{r} K$ with $r>0$. Since for $b_{1}, b_{2} \in \mathbf{Z}_{2}^{*}$ we can find $b_{1}^{\prime}, b_{2}^{\prime} \in \mathbf{Z}_{2}^{*}$ such that $2 b_{1}^{\prime} \perp b_{2}^{\prime} \cong 2 b_{1} \perp b_{2}$ and $-b_{2}^{\prime} b_{3} \equiv 1 \bmod 4$, we may assume that $r \geq 2$ if $s=0$ in the first two cases. Then by Theorem 2.1 , we have

$$
\begin{aligned}
\alpha_{2}\left(4^{i} B_{1} \perp b_{3}\right)=2^{4-2 k} \sum_{X \in \mathcal{X}_{2}^{\prime}} \alpha_{2}\left(4^{i} B_{1}[X] \perp b_{3}\right) & \\
& -2^{9-4 k} \alpha_{2}\left(4^{i-1} B_{1} \perp b_{3}\right)+\beta_{2}\left(4^{i} B_{1} \perp b_{3}\right)
\end{aligned}
$$

for $i=0,1$, where $\mathcal{X}_{2}^{\prime}$ is the set defined in the proof of Proposition 3.2.2. Thus we have

$$
\begin{aligned}
\tilde{\gamma}\left(B_{1}, b_{3}\right)=2^{4-2 k} \sum_{X \in \mathcal{X}_{2}^{\prime}} \tilde{\gamma}\left(B_{1}[X], b_{3}\right)-2^{9-4 k} \tilde{\gamma}\left(4^{-1} B_{1}, b_{3}\right) & \\
& +d\left(1-2^{4-2 k}\right)\left(1-2^{5-2 k}\right) .
\end{aligned}
$$

Thus by the induction hypothesis the assertion holds for the first two cases. The assertion also holds for the case $B_{1}=2^{r} K$ with $r>0$. Similarly the assertion can be proved for $p \neq 2$.

The following corollary can be easily derived from the induction formula in Theorem 3.2.1.

Corollary to Theorem 3.2.1. For the matrix $B$ in Theorem 3.2.1, let $m_{2}=m_{2 p}(B), m_{3}=m_{3 p}(B)$ be the invariants defined in Section 1. Then we have

$$
\begin{aligned}
& \alpha_{p}(B)=\alpha_{p}\left(B\left[p^{-\left[\left(m_{2}-2 \delta_{2 p}\right) / 2\right]} E_{2} \perp 1\right]\right) p^{(5-2 k)\left(\left[m_{2} / 2\right]-\delta_{2 p}\right)} \\
& +d \eta_{p}(B) p^{(2-k) m_{3}-(3-2 k)\left(\left[m_{2} / 2\right]-\delta_{2 p}-1\right)} \sum_{i=0}^{\left[m_{2} / 2\right]-\delta_{2 p}-1} p^{(3-2 k) i}+d \sum_{i=0}^{\left[m_{2} / 2\right]-\delta_{2 p}-1} p^{(5-2 k) i}
\end{aligned}
$$

where $E_{2}$ denotes the unit matrix of degree 2.

\subsection{Third step}

For a non-degenerate symmetric matrix $B$ of degree 3 with entries in $\mathbf{Q}_{p}$, put

$$
\gamma(B)=\alpha_{p}(p B)-\left(p^{5-2 k}+p^{3-k}\right) \alpha_{p}(B)+p^{8-3 k} \alpha_{p}\left(p^{-1} B\right) .
$$


We define a subset $\mathcal{H}_{3}^{\prime}\left(\mathbf{Z}_{2}\right)$ of $S_{3}\left(\mathbf{Q}_{2}\right)$ by

$$
\begin{aligned}
& \mathcal{H}_{3}^{\prime}\left(\mathbf{Z}_{2}\right)=\left\{B ; B \cong 2^{r} b_{1} \perp b_{2} \perp 2^{-1} b_{3} \text { with } r \geq 0, b_{1}, b_{2}, b_{3} \in \mathbf{Z}_{2}^{*}\right\} \\
& \cup\left\{B ; B \cong 2^{r} b_{1} \perp 2^{-1} b_{2} \perp 2^{-1} b_{3}\right. \\
& \left.\quad \text { with } r \geq 0, b_{1}, b_{2}, b_{3} \in \mathbf{Z}_{2}^{*},-b_{2} b_{3} \not \equiv 1 \bmod 4\right\} \\
& \cup\left\{B ; B \cong 2^{r} K \perp 2^{s} b_{3}\right. \\
& \left.\quad \text { with } b_{3} \in \mathbf{Z}_{2}^{*},(r, s)=(0,-1),(-1,0),(0,0), K=H, Y\right\} .
\end{aligned}
$$

We remark that any element of $\mathcal{H}_{3}\left(\mathbf{Z}_{2}\right) \cap \mathcal{H}_{3}^{\prime}\left(\mathbf{Z}_{2}\right)$ is equivalent over $\mathbf{Z}_{2}$ to $b_{1} \perp K$.

THEOREM 3.3.1. Let $B \in \mathcal{H}_{3}\left(\mathbf{Z}_{p}\right) \cup \mathcal{H}_{3}^{\prime}\left(\mathbf{Z}_{p}\right)$. Here we understand $\mathcal{H}_{3}^{\prime}\left(\mathbf{Z}_{p}\right)$ is the empty set if $p \neq 2$. Then we have

$$
\gamma(B)=d\left(1+p^{(2-k)\left(m_{3}+3\right)} \eta_{p}(B)\right)
$$

where $m_{3}=m_{3 p}(B)$.

To prove the theorem first we have

Lemma 3.3.2. For $B \in \mathcal{H}_{3}^{\prime}\left(\mathbf{Z}_{2}\right)$, the assertion in Theorem 3.3 .1 is true.

Proof. The assertion follows from Theorem 3.1.3 except for $b_{1} \perp K$. If $B=b_{1} \perp K$ then we have $b_{1}^{\prime} \perp H$ for some $b_{1}^{\prime} \in \mathbf{Z}_{2}^{*}$, and therefore we may assume $K=H$. Then by (1) of Proposition 2.2, Proposition 2.3, and Theorems 2.1 we have

$$
\alpha_{2}(2 B)=2^{4-2 k} \alpha_{2}\left(H \perp b_{3}\right)+d\left(1-2^{4-2 k}\right)\left(1+2^{3-k}\right) \text { and } \alpha_{2}(B)=d .
$$

Thus again by Proposition 2.3 the assertion holds.

Proof of Theorem 3.3.1. The assertion for $p \neq 2$ has been proved in [Ki2], and thus we treat only the case $p=2$. But we remark that the case for $p \neq 2$ can be proved similarly to the case of $p=2$ by using our method. We prove the theorem by induction on the order defined in Section 2 in $\mathcal{H}_{3}\left(\mathbf{Z}_{2}\right) \cup \mathcal{H}_{3}^{\prime}\left(\mathbf{Z}_{2}\right)$. Clearly any maximal element of $\mathcal{H}_{3}\left(\mathbf{Z}_{2}\right) \cup \mathcal{H}_{3}^{\prime}\left(\mathbf{Z}_{2}\right)$ belongs to $\mathcal{H}_{3}^{\prime}\left(\mathbf{Z}_{2}\right)$. Thus by Lemma 3.3.2 the assertion holds for all maximal elements in $\mathcal{H}_{3}\left(\mathbf{Z}_{2}\right) \cup \mathcal{H}_{3}^{\prime}\left(\mathbf{Z}_{2}\right)$. Assume that $B$ is not maximal and the assertion holds for any $B^{\prime}$ such that $B<B^{\prime}$. The proof is divided into four 
cases:

(1) $B \in \mathcal{H}_{3}^{\prime}\left(\mathbf{Z}_{2}\right) \quad(2) B \in \mathcal{H}_{3}\left(\mathbf{Z}_{2}\right)$ but $2^{-1} B \notin \mathcal{H}_{3}\left(\mathbf{Z}_{2}\right) \quad$ (3) $2^{-1} B \in$ $\mathcal{H}_{3}\left(\mathbf{Z}_{2}\right)$ but $2^{-2} B \notin \mathcal{H}_{3}\left(\mathbf{Z}_{2}\right)(4) 2^{-2} B \in \mathcal{H}_{3}\left(\mathbf{Z}_{2}\right)$.

Case (1) The assertion holds by Lemma 3.3.2.

From now on let $b_{1}, b_{2}, b_{3} \in \mathbf{Z}_{2}^{*}$ and $K=H$ or $Y$.

Case (2) In view of Lemma 3.3.2 we may assume $B \not b_{1} \perp K$. Thus in view of the remark after Proposition 2.2 we may assume $B=2^{s} K \perp b_{3}$ with $s>0,2^{r} b_{1} \perp 2^{s} b_{2} \perp b_{3}$ with $r \geq s>0,2^{r} b_{1} \perp b_{2} \perp b_{3}$ with $r>0$, or $2^{r} b_{1} \perp K$ with $r>0$.

First let $B=2^{s} K \perp b_{3}$ with $s>0$ or $B=2^{r} b_{1} \perp 2^{s} b_{2} \perp b_{3}$ with $r \geq s>0$. Then by Theorem 2.1, we have

$$
\begin{aligned}
\alpha_{2}\left(2^{i} B\right)=2^{4-2 k} \alpha_{2}\left(2^{i} B\left[f_{1}\right]\right)+2^{4-2 k} \sum_{\alpha=0,1} \alpha_{2}\left(2^{i} B\left[f_{2}(\alpha)\right]\right) \\
\\
\quad-2^{9-4 k} \alpha_{2}\left(2^{i} B\left[g_{1}\right]\right)+\beta_{2}\left(2^{i} B\right)
\end{aligned}
$$

for $i=1,0$. Further by Proposition 2.3 we have

$$
\beta_{2}(B)=d\left(1-2^{4-2 k}\right), \quad \beta_{2}(2 B)=d\left(1-2^{4-2 k}\right)\left(1+2^{3-k}\right) .
$$

Thus we have

$$
\begin{aligned}
\gamma(B)=2^{4-2 k} \gamma\left(B\left[f_{1}\right]\right)+2^{4-2 k} & \sum_{\alpha=0,1} \gamma\left(B\left[f_{2}(\alpha)\right]\right) \\
& -2^{9-4 k} \gamma\left(B\left[g_{1}\right]\right)+d\left(1-2^{4-2 k}\right)\left(1-2^{5-2 k}\right) .
\end{aligned}
$$

By assumption all the $B\left[f_{1}\right], B\left[f_{2}(0)\right], B\left[f_{2}(1)\right]$, and $B\left[g_{1}\right]$ belong to $\mathcal{H}_{3}\left(\mathbf{Z}_{2}\right)$ $\cup \mathcal{H}_{3}^{\prime}\left(\mathbf{Z}_{2}\right)$. Thus by the induction hypothesis and the case (1) we have

$$
\begin{aligned}
\gamma(B)= & 2^{4-2 k} d\left(1+2^{(2-k)\left(m_{3}+1\right)} \eta_{2}(B)\right)+2^{5-2 k} d\left(1+2^{(2-k)\left(m_{3}+1\right)} \eta_{2}(B)\right) \\
& \quad-2^{9-4 k} d\left(1+2^{(2-k)\left(m_{3}-1\right)} \eta_{2}(B)\right)+d\left(1-2^{4-2 k}\right)\left(1-2^{5-2 k}\right) \\
= & d\left(1+2^{(2-k)\left(m_{3}+3\right)} \eta_{2}(B)\right) .
\end{aligned}
$$

Next let $B=2^{r} b_{1} \perp b_{2} \perp b_{3}$ with $r>0$. Then by Theorem 2.1, we have

$$
\begin{aligned}
\alpha_{2}\left(2^{i} B\right)=2^{4-2 k} \alpha_{2}\left(2^{i} B\left[f_{1}\right]\right)+2^{4-2 k} & \sum_{\alpha=0,1} \alpha_{2}\left(2^{i} B\left[f_{3}(\alpha, 1)\right]\right) \\
& -2^{9-4 k} \alpha_{2}\left(2^{i} B\left[g_{2}(1)\right]\right)+\beta_{2}\left(2^{i} B\right) .
\end{aligned}
$$

Further by Proposition 2.3 we have

$$
\beta_{2}(B)=d\left(1-2^{4-2 k}\right), \beta_{2}(2 B)=d\left(1-2^{4-2 k}\right)\left(1+2^{3-k}\right) .
$$


Thus we have

$$
\begin{aligned}
\gamma(B)=2^{4-2 k} \gamma\left(B\left[f_{1}\right]\right)+2^{4-2 k} & \sum_{\alpha=0,1} \gamma\left(B\left[f_{2}(\alpha)\right]\right) \\
& -2^{9-4 k} \gamma\left(B\left[g_{1}\right]\right)+d\left(1-2^{4-2 k}\right)\left(1-2^{5-2 k}\right) .
\end{aligned}
$$

Thus the assertion can be proved similarly to the first case.

Thirdly let $B=2^{r} b_{1} \perp H$ with $r>0$. Then by Theorem 2.1, we have

$$
\begin{aligned}
& \alpha_{2}(2 B)=2^{4-2 k} \alpha_{2}\left(2 B\left[f_{1}\right]\right) \\
& +2^{4-2 k} \sum_{\alpha=0,1} \alpha_{2}\left(2 B\left[f_{2}(\alpha)\right]+2^{4-2 k} \sum_{\alpha=0,1} \alpha_{2}\left(2 B\left[f_{2}(\alpha, 0)\right]\right)\right. \\
& \quad-2^{9-4 k} \alpha_{2}\left(2 B\left[g_{1}\right]\right)-2^{9-4 k} \alpha_{2}\left(2 B\left[g_{2}(0)\right]\right)+\beta_{2}(2 B),
\end{aligned}
$$

and

$$
\alpha_{2}(B)=2^{4-2 k} \alpha_{2}\left(B\left[f_{1}\right]\right)+\beta_{2}(B) .
$$

By easy calculation we have

$$
2 B\left[f_{2}(\alpha)\right] \cong 2^{r+1} b_{1}^{\prime}(\alpha) \perp H, \quad 2 B\left[f_{2}(\alpha, 0)\right] \cong 2^{r+1} b_{1}^{\prime \prime}(\alpha) \perp H,
$$

and

$$
2 B\left[g_{1}\right]=2 B\left[g_{2}(0)\right]=2^{r-1} b_{1} \perp H,
$$

where $b_{1}^{\prime}(\alpha), b_{1}^{\prime \prime}(\alpha) \in \mathbf{Z}_{2}^{*}$. Further by Proposition 2.3 we have

$$
\beta_{2}(B)=d\left(1+2^{2-k}\right), \quad \beta_{2}(2 B)=d\left(1-2^{4-2 k}\right)\left(1+2^{3-k}\right) .
$$

Thus the assertion can be proved by $(*)$ in the proof of (1.1) of Lemma 3.1.2.

Finally let $B=2^{r} b_{1} \perp Y$ with $r>0$. Then by Theorem 2.1, we have

$$
\alpha_{2}\left(2^{i} B\right)=2^{4-2 k} \alpha_{2}\left(2^{i} B\left[f_{1}\right]\right)+\beta_{2}\left(2^{i} B\right)
$$

for $i=0,1$. Further by Proposition 2.3 we have

$$
\beta_{2}(B)=d\left(1-2^{2-k}\right), \quad \beta_{2}(2 B)=d\left(1-2^{4-2 k}\right)\left(1+2^{3-k}\right) .
$$

Thus the assertion holds.

Case (3) It suffices to prove the assertion for the case $B=2^{r} b_{1} \perp 2^{s} b_{2} \perp 2 b_{3}$ with $r \geq s \geq 1,2^{r} K \perp 2 b_{3}$ with $r \geq 1$ or $B=2^{r} b_{1} \perp 2 K$ with $r \geq 1$. 
First let $B_{1}=2^{r} K$ with $r>1$ or $B_{1}=2^{r} b_{1} \perp 2^{s} b_{2}$ with $r \geq s>1$, and put $B=B_{1} \perp 2 b_{3}$. Then by Theorem 2.1, we have

$$
\begin{aligned}
\alpha_{2}\left(2^{i} B\right)=R\left(2^{i} B\right)+2^{4-2 k} \alpha_{2}\left(2^{i} B\left[f_{1}\right]\right)+2^{4-2 k} \sum_{\alpha=0,1} \alpha_{2}\left(2^{i} B\left[f_{2}(\alpha)\right]\right) \\
-2^{9-4 k} \alpha_{2}\left(B\left[g_{1}\right]\right)+\beta_{2}\left(2^{i}\left(2^{r} b_{1} \perp 2^{s} b_{2} \perp b_{3}\right)\right)
\end{aligned}
$$

for $i=1,0,-1$, where

$$
\begin{aligned}
R\left(2^{i} B\right)=2^{4-2 k} \sum_{\alpha, \beta=0,1} \alpha_{2}\left(2 B\left[f_{3}(\alpha, \beta)\right]\right)-2^{9-4 k} \sum_{\alpha=0,1} \alpha_{2}\left(2 B\left[g_{2}(\alpha)\right]\right) \\
-2^{9-4 k} \sum_{\alpha, \beta=0,1} \alpha_{2}\left(2 B\left[g_{3}(\alpha, \beta)\right]\right)+2^{15-6 k} \alpha_{2}\left(2^{-1} B\right),
\end{aligned}
$$

or 0 according as $i=1$ or not. Put $\tilde{B}(\alpha, \beta)=2 B\left[f_{3}(\alpha, \beta)\right]$ for any $\alpha, \beta=0$, 1. Clearly we have

$$
\begin{aligned}
g_{2}(\beta) \equiv f_{3}(\alpha, \beta) f_{1}, g_{3}\left(\alpha^{\prime}, \alpha\right) \equiv f_{3}(\alpha, \beta) f_{2}\left(\alpha^{\prime}\right) & \\
& \text { and } 2^{-1} E_{3} \equiv f_{3}(\alpha, \beta) g_{1} \bmod \Gamma
\end{aligned}
$$

for any $\alpha, \beta, \alpha^{\prime}=0,1$. Thus we have

$$
\left.2 B\left[g_{2}(\beta)\right] \cong \tilde{B}(\alpha, \beta)\left[f_{1}\right], \quad 2 B\left[g_{3}\left(\alpha^{\prime}, \alpha\right)\right] \cong \tilde{B}(\alpha, \beta)\left[f_{2}\left(\alpha^{\prime}\right)\right]\right)
$$

and

$$
\left.2 B\left[2^{-1} E_{3}\right]\right] \cong \tilde{B}(\alpha, \beta)\left[g_{1}\right]
$$

for any $\alpha, \beta, \alpha^{\prime}=0,1$. By easy calculation for any $\alpha, \beta=0,1$ we have

$$
\tilde{B}(\alpha, \beta) \cong 2 B_{1}(\alpha, \beta) \perp b_{3}^{\prime}(\alpha, \beta)
$$

with $B_{1}(\alpha, \beta) \in 2 \mathcal{H}_{2}\left(\mathbf{Z}_{2}\right) \cap G L_{2}\left(\mathbf{Q}_{2}\right)$ and $b_{3}^{\prime}(\alpha, \beta) \in \mathbf{Z}_{2}^{*}$. On the other hand we have $\tilde{B}(\alpha, \beta)[X] \notin \mathcal{H}_{3}\left(\mathbf{Z}_{2}\right)$ for $\alpha, \beta=0,1$ and $X \in \mathcal{E} \backslash\left\{f_{1}, f_{2}\left(\alpha^{\prime}\right)\left(\alpha^{\prime}=\right.\right.$ $\left.0,1), g_{1}\right\}$. Thus Theorem 2.1 and Proposition 2.3 imply

$$
R(2 B)=2^{4-2 k} \sum_{\alpha, \beta=0,1} \beta(\tilde{B}(\alpha, \beta))=2^{6-2 k} d\left(1-2^{4-2 k}\right) .
$$

Thus by the induction hypothesis and Proposition 2.3 we have

$$
\begin{aligned}
\gamma(B) & =R(2 B)+2^{4-2 k} \gamma\left(B\left[f_{1}\right]\right)+2^{4-2 k} \sum_{\alpha=0,1} \gamma\left(B\left[f_{2}(\alpha)\right]\right)-2^{9-4 k} \gamma\left(B\left[g_{1}\right]\right) \\
& +\beta_{2}(2 B)-\left(2^{3-k}+2^{5-2 k}\right) \beta_{2}(B)+2^{8-3 k} \beta_{2}\left(2^{-1} B\right) \\
& =d\left(1+2^{(2-k)\left(m_{3}+3\right)} \eta_{2}(B)\right) .
\end{aligned}
$$


Next let $B=2^{r} b_{1} \perp 2 b_{2} \perp 2 b_{3}$ with $r>1$. Then Theorem 2.1 implies

$$
\begin{aligned}
\alpha_{2}\left(2^{i} B\right)=R\left(2^{i} B\right)+2^{4-2 k} \alpha_{2}\left(2^{i} B\left[f_{1}\right]\right) & +2^{4-2 k} \sum_{\alpha=0,1} \alpha_{2}\left(2^{i} B\left[f_{3}(\alpha, 1)\right]\right) \\
& -2^{9-4 k} \alpha_{2}\left(2^{i} B\left[g_{2}(1)\right]\right)+\beta_{2}\left(2^{i} B\right)
\end{aligned}
$$

for $i=1,0,-1$, where

$$
\begin{aligned}
R\left(2^{i} B\right)=2^{4-2 k} \sum_{\alpha=0,1} & \alpha_{2}\left(2 B\left[f_{2}(\alpha)\right]\right)+2^{4-2 k} \sum_{\alpha=0,1} \alpha_{2}\left(2 B\left[f_{3}(\alpha, 0)\right]\right) \\
& -2^{9-4 k} \alpha_{2}\left(2 B\left[g_{2}(0)\right]\right)-2^{9-4 k} \alpha_{2}\left(2 B\left[g_{1}\right]\right) \\
& -2^{9-4 k} \sum_{\alpha, \beta=0,1} \alpha_{2}\left(2 B\left[g_{3}(\alpha, \beta)\right]\right)+2^{15-6 k} \alpha_{2}\left(2^{-1} B\right),
\end{aligned}
$$

or 0 according as $i=1$ or not. Similarly to the above case, Theorem 2.1 yields

$$
R(2 B)=2^{4-2 k} \sum_{\alpha=0,1} \beta_{2}\left(2 B\left[f_{2}(\alpha)\right]\right)+2^{4-2 k} \sum_{\alpha=0,1} \beta_{2}\left(2 B\left[f_{3}(\alpha, 0)\right]\right) .
$$

On the other hand, easy calculation shows

$$
2 B\left[f_{2}(\alpha)\right] \cong B_{1}^{\prime}(\alpha) \perp b_{3}^{\prime}(\alpha), \quad 2 B\left[f_{3}(\alpha, 0)\right] \cong B_{1}^{\prime \prime}(\alpha) \perp b_{3}^{\prime \prime}(\alpha),
$$

where $B_{1}^{\prime}(\alpha), B_{1}^{\prime \prime}(\alpha) \in 2 \mathcal{H}_{2}\left(\mathbf{Z}_{2}\right) \cap G L_{2}\left(\mathbf{Q}_{2}\right)$ and $b_{3}^{\prime}(\alpha), b_{3}^{\prime \prime}(\alpha) \in \mathbf{Z}_{2}^{*}$. Thus by Proposition 2.3 we have

$$
R(2 B)=2^{6-2 k} d\left(1-2^{4-2 k}\right) .
$$

Thus the assertion holds.

The rest of the cases can be treated in the same way.

Case (4) Theorem 2.1 tells us

$$
\begin{aligned}
\alpha_{2}\left(2^{i} B\right)=2^{4-2 k} \sum_{f \in \mathcal{F}} \alpha_{2}\left(2^{i} B[f]\right)-2^{9-4 k} & \sum_{g \in \mathcal{G}} \alpha_{2}\left(2^{i} B[g]\right) \\
& +2^{15-6 k} \alpha_{2}\left(2^{-2+i} B\right)+\beta_{2}\left(2^{i} B\right),
\end{aligned}
$$

for $i=-1,0,1$. By assumption $B[X] \in \mathcal{H}_{3}\left(\mathbf{Z}_{2}\right)$ for any $X \in \mathcal{E}$. Thus by the induction hypothesis, the case (3), and Proposition 2.3 we have

$$
\gamma(B)=7 \times 2^{4-2 k} d\left(1+2^{(2-k)\left(m_{3}+1\right)} \eta_{2}(B)\right)
$$




$$
\begin{aligned}
& \quad-7 \times 2^{9-4 k} d\left(1+2^{(2-k)\left(\left(m_{3}-1\right)\right.} \eta_{2}(B)\right) \\
& \quad+2^{15-6 k} d\left(1+2^{(2-k)\left(m_{3}-3\right)} \eta_{2}(B)\right) \\
& \quad+d\left(1-2^{4-2 k}\right)\left(1+2^{3-k}\right)\left(1-2^{3-k}-2^{5-2 k}+2^{8-3 k}\right) \\
& =d\left(1+2^{(2-k)\left(m_{3}+3\right)} \eta_{2}(B)\right) .
\end{aligned}
$$

Thus the assertion holds by induction.

By the induction formula in Theorem 3.3.1 we easily see

COROLlaRY. The notation being as above, we have

$$
\begin{array}{r}
\alpha_{p}(B)=\alpha_{p}\left(p^{-m_{1}} B\right) p^{(3-k) m_{1}} \sum_{j=0}^{m_{1}} p^{(2-k) j}+d \sum_{i=0}^{m_{1}-1} \sum_{j=0}^{m_{1}-1-i} p^{(5-2 k) j} p^{(3-k) i} \\
+d \eta_{p}(B) p^{(2-k) m_{3}-(3-2 k)\left(m_{1}-1\right)} \sum_{i=0}^{m_{1}-1} \sum_{j=0}^{m_{1}-1-i} p^{(3-2 k) j} p^{(2-k) i}
\end{array}
$$

where $m_{1}=m_{1 p}(B)$.

\subsection{Final step}

Now in this subsection we complete the proof of Theorem 1.2. Let $m_{1}=m_{1 p}(B), m_{2}=m_{2 p}(B), m_{3}=m_{3 p}(B), \eta=\eta_{p}(B), \xi=\xi_{p}(B)$, $n^{\prime}=n_{p}^{\prime}(B)$ be as in Section 1 .

First let $B=p^{m_{1}}\left(p^{r} B_{1} \perp b_{3}\right)$ with $r \geq 0, B_{1} \in \mathcal{H}_{2}\left(\mathbf{Z}_{p}\right), b_{3} \in \mathbf{Z}_{p}^{*}$. Then by Theorem 3.1.3 and Corollary to Theorem 3.2.1 we have

$$
\begin{aligned}
& \alpha_{p}\left(p^{-m_{1}} B\right)=d\left\{a\left(B_{1}\right)\right. \\
& \left.+p^{(5-2 k) \delta_{2 p}} \sum_{i=0}^{m_{3}-2 m_{2}+m_{1}} p^{(2-k) i} \xi^{i+2}\right\} p^{(5-2 k)\left(\left[m_{2} / 2\right]-m_{1}-\delta_{2 p}\right)} \\
& +d \eta p^{(2-k)\left(m_{3}-3 m_{1}\right)-(3-2 k)\left(\left[m_{2} / 2\right]-m_{1}-\delta_{2 p}-1\right)} \sum_{i=0}^{\left[m_{2} / 2\right]-m_{1}-\delta_{2 p}-1} p^{(3-2 k) i} \\
& +d \sum_{i=0}^{\left[m_{2} / 2\right]-m_{1}-\delta_{2 p}-1} p^{(5-2 k) i},
\end{aligned}
$$

where

$$
a\left(B_{1}\right)= \begin{cases}0 & \text { if } p \neq 2 \text { and } m_{2} \text { is even } \\ 1 & \text { if } p=2, m_{2} \text { is even and } B_{1}=K \\ 1+p^{\left(m_{3}-4\left[m_{2} / 2\right]+m_{1}+4 \delta_{2 p}\right)(2-k)} \eta & \text { otherwise. }\end{cases}
$$


Thus we have

$$
\begin{aligned}
& \alpha_{p}\left(p^{-m_{1}} B\right)=d \sum_{j=0}^{\left[m_{2} / 2\right]-m_{1}-\delta_{2 p}-m^{\prime}} p^{(5-2 k) j} \\
& +d p^{(2-k) m_{3}-(3-2 k)\left(\left[m_{2} / 2\right]-\delta_{2 p}\right)-(3-k) m_{1}} \eta \sum_{j=n^{\prime}}^{\left[m_{2} / 2\right]-m_{1}-\delta_{2 p}} p^{(3-2 k) j} \\
& +d p^{(5-2 k)\left(\left[m_{2} / 2\right]-m_{1}\right)} \sum_{i=0}^{m_{3}-2 m_{2}+m_{1}} p^{(2-k) i} \xi^{i+2}
\end{aligned}
$$

where $m^{\prime}=m_{p}^{\prime}(B)=1$ or 0 according as $p \neq 2$ and $m_{2}$ is even, or not. Clearly we have $\left[m_{2} / 2\right]-\delta_{2 p}-m^{\prime}=\left[\left(m_{2}-\delta_{2 p}-1\right) / 2\right]$. Thus the assertion can be derived from Corollary to Theorem 3.3.1 by remarking that we have

$$
\begin{array}{r}
d \sum_{j=0}^{\left[m_{2} / 2\right]-m_{1}-\delta_{2 p}-m^{\prime}} p^{(5-2 k) j} p^{(3-k) m_{1}} \sum_{i=0}^{m_{1}} p^{(2-k) i}+d \sum_{i=0}^{m_{1}-1}\left(\sum_{j=0}^{m_{1}-1-i} p^{(5-2 k) j}\right) p^{(3-k) i} \\
\left.=d \sum_{i=0}^{m_{1}} \sum_{j=0}^{\left[m_{2} / 2\right]-\delta_{2 p}-m^{\prime}-i} p^{(5-2 k) j}\right) p^{(3-k) i}
\end{array}
$$

and

$$
\begin{gathered}
d \eta p^{(2-k) m_{3}-(3-2 k)\left(\left[m_{2} / 2\right]-\delta_{2 p}\right)-(3-k) m_{1}} \sum_{j=n^{\prime}}^{\left[m_{2} / 2\right]-\delta_{2 p}-m_{1}} p^{(3-2 k) j} p^{(3-k) m_{1}} \sum_{i=0}^{m_{1}} p^{(2-k) i} \\
+d \eta p^{(2-k) m_{3}-(3-2 k)\left(m_{1}-1\right)} \sum_{i=0}^{m_{1}-1}\left(\sum_{j=0}^{m_{1}-1-i} p^{(3-2 k) j}\right) p^{(2-k) i} \\
=d \eta p^{m_{3}(2-k)-(3-2 k)\left(\left[m_{2} / 2\right]-\delta_{2 p}\right)} \sum_{i=0}^{m_{1}}\left(\sum_{j=n^{\prime}}^{\left[m_{2} / 2\right]-\delta_{2 p}-i} p^{(3-2 k) i}\right) p^{(2-k) i}
\end{gathered}
$$

Next let $p=2$ and $B=2^{m_{1}}\left(2^{r} b_{1} \perp K\right)$. Then by Theorem 3.1.3, we have

$$
\alpha_{2}\left(2^{-m_{1}} B\right)=d \sum_{i=0}^{m_{3}-2 m_{2}+m_{1}}\left(2^{2-k} \xi\right)^{i} .
$$

Thus the assertion also holds in this case. 


\section{§4. Comments}

We give Theorem 1.2 in more concise form. First let $p \neq 2$. Then a nondegenerate symmetric matrix $B$ of degree 3 with entries in $\mathbf{Z}_{p}$ is equivalent, over $\mathbf{Z}_{p}$, to a matrix of the form (4) in Section 1 . Then we have

$$
\begin{gathered}
d^{-1} \alpha_{p}(B)=\sum_{i=0}^{t}\left(\sum_{j=0}^{[(s+t-1) / 2]-i} p^{(5-2 k) j}\right) p^{(3-k) i} \\
+\left(-p^{r-t} b_{1} b_{3},-p^{s-t} b_{2} b_{3}\right)_{p} p^{(2-k)(r+s+t)-(3-2 k)[(s+t) / 2]} \sum_{i=n^{\prime}}^{t} \sum_{j=0}^{[(s+t) / 2]-i} p^{(3-2 k) j} p^{(2-k) i} \\
+u(B),
\end{gathered}
$$

where $n^{\prime}=1$ or 0 according as $s-t$ is even or odd, and

$$
u(B)=p^{(5-2 k)(s+t) / 2-(2-k) t} \sum_{i=0}^{r-s}\left(p^{2-k} \chi_{p}\left(-p^{s-t} b_{2} b_{3}\right)\right)^{i} \sum_{j=0}^{t} p^{(2-k) j} \text { or } 0
$$

according as $s-t$ is even or odd. This coincides with [Ki 2, Theorem].

Next let $p=2$. Then a non-degenerate half-integral matrix $B$ of degree 3 over $\mathbf{Z}_{2}$ is equivalent, over $\mathbf{Z}_{2}$, to one of the forms (1), (2) and (3) in Section 1 .

(1) The case where $B$ is equivalent to a matrix of the type (1):

$$
\begin{aligned}
& d^{-1} \alpha_{2}(B)=\sum_{i=0}^{t-1}\left(\sum_{j=0}^{t-1-i} 2^{(5-2 k) j}\right) 2^{(3-k) i} \\
& +\chi_{2}(-\operatorname{det} K)^{s-t} 2^{(2-k)(s+2 t)-(3-2 k)(t-1)} \sum_{i=0}^{t}\left(\sum_{j=0}^{t-1-i} 2^{(3-2 k) j}\right) 2^{(2-k) i} \\
& +2^{(3-k) t} \sum_{i=0}^{s-t}\left(\chi_{2}(-\operatorname{det} K) 2^{2-k}\right)^{i} \sum_{j=0}^{t} 2^{(2-k) j}
\end{aligned}
$$

(2) The case where $B$ is equivalent to a matrix of the type (2):

$$
\begin{aligned}
& d^{-1} \alpha_{2}(B)=\sum_{i=0}^{t}\left(\sum_{j=0}^{[(s+t) / 2]-i} 2^{(5-2 k) j}\right) 2^{(3-k) i} \\
& +\chi_{2}(-\operatorname{det} K)^{s-t} 2^{(2-k)(2 s+t)-(3-2 k)[(s+t) / 2]} \sum_{i=0}^{t}\left(\sum_{j=n^{\prime}}^{[(s+t) / 2]-i} 2^{(3-2 k) j}\right) 2^{(2-k) i},
\end{aligned}
$$


where $n^{\prime}=1$ or 0 according as $s-t$ is even or odd.

(3) The case where $B$ is equivalent to a matrix of the type (3):

$$
\begin{aligned}
d^{-1} \alpha_{2}(B)= & \sum_{i=0}^{t}\left(\sum_{j=0}^{[(s+t) / 2]-i} 2^{(5-2 k) j}\right) 2^{(3-k) i} \\
+\left(-2^{r-t} b_{1} b_{3},-2^{s-t} b_{2} b_{3}\right){ }_{2} 2^{(2-k)(r+s+t+2)-(3-2 k)[(s+t) / 2]} & \\
& \times \sum_{i=0}^{t} \sum_{j=0}^{[(s+t) / 2]-i} 2^{(3-2 k) j} 2^{(2-k) i}+u(B)
\end{aligned}
$$

where

$$
u(B)=2^{(5-2 k)[(s+t+2) / 2]-(2-k) t} \sum_{i=0}^{r-s-2} \xi(B)^{i+2} 2^{(2-k) i} \sum_{j=0}^{t-1} 2^{(2-k) j} \text { or } 0
$$

according as $s-t$ is even or odd.

\section{REFERENCES}

[Ka1] H. Katsurada, A certain formal power series of several variables attached to local densities of quadratic forms I, J. Number Theory, 51 (1995), 169-209.

[Ka2] H. Katsurada, A certain formal power series of several variables attached to local densities of quadratic forms II, Ser. A, Proc. Japan Acad., 70 (1994), 208-211.

[Ki1] Y. Kitaoka, A note on local densities of quadratic forms, Nagoya Math. J., 92 (1983), 145-152.

[Ki2] Y. Kitaoka, Fourier coefficients of Eisenstein series of degree 3, Ser. A, Proc. Japan Acad., 60 (1984), 259-261.

[Ki3] Y. Kitaoka, Dirichlet series in the theory of quadratic forms, Nagoya Math., 92 (1984), 73-84.

[Ki4] Y. Kitaoka, Arithmetic of quadartic forms, Cambridge. Tracts Math., 106 (1993), Cambridge Univ. Press, Cambridge.

[Ma1] H. Maaß, Über Fourierkoeffizienten der Eisensteinreihen zweiten Grades, Mat. Fys. Medd. Dan. Vid. Selsk., 34 (1964), 1-25.

[Ma2] H. Maaß, Die Fourierkoeffizienten der Eisensteinreihen zweiten Grades, ibid., 38 (1972), 1-13.

[Mi] T. Miyake, Modular forms, Springer-Verlag, Berlin Heiderberg, 1989.

[O-W] M. Ozeki, T. Washio, Explicit formulas for the Fourier coefficients of Eisenstein series of degree 3, J. reine angew. Math., 345 (1983), 148-171.

Muroran Institute of Technology

27-1 Mizumoto Muroran 050

Japan

hidenori@muroran-it.ac.jp 